
\title{
25 Research Square \\ Performance Evaluation of GAA Nanosheet FET with Varied Geometrical and Process Parameters
}

Aruna Kumari Neelam ( $\nabla$ arunavignan4@gmail.com )

National Institute of Technology Warangal

Prithvi P

National Institute of Technology Warangal

\section{Research Article}

Keywords: Nanosheet, GAA, SCEs, Process Variations, Scaling, Sub -7 nm

Posted Date: October 14th, 2021

DOI: https://doi.org/10.21203/rs.3.rs-965209/v1

License: (c) (i) This work is licensed under a Creative Commons Attribution 4.0 International License. Read Full License 


\section{Abstract}

Nanosheet Field Effect Transistor (NSFET) is a viable contender for future scaling in sub-7-nm technology. This paper provides insights into the variations of DC FOMs for different geometrical configurations of the NSFET. In this script, DC performance of 3D GAA NSFET is analyzed by varying the width, thickness of the device. Moreover, the gate length is scaled from $20 \mathrm{~nm}$ to $5 \mathrm{~nm}$ to check for the device suitability in logic applications. The thickness and width of each nanosheet are varied in the range of 5 to $9 \mathrm{~nm}$, and 10 to $50 \mathrm{~nm}$ respectively to analyse the performance dependency on the geometry of the device. The impact of geometry of NSFET on various DC performance metrics like transfer characteristics, sub-threshold swing (SS), on current $\left(\mathrm{I}_{\mathrm{ON}}\right)$, off current $\left(\mathrm{I}_{\mathrm{OFF}}\right)$, switching ratio $\left(\mathrm{I}_{\mathrm{ON}} / \mathrm{I}_{\mathrm{OFF}}\right)$, threshold voltage $\left(\mathrm{V}_{\mathrm{th}}\right)$ and drain induced barrier lowering (DIBL) are studied. On top of that, the device's electrical characteristics are analyzed for a wide range of temperatures from $-43^{\circ} \mathrm{C}$ to $127^{\circ} \mathrm{C}$ to identify the temperature compensation point and is observed at $\mathrm{V}_{\mathrm{GS}}=0.55 \mathrm{~V}$ and $\mathrm{I}_{\mathrm{D}}=3.86 \times 10^{-6} \mathrm{~A}$. Furthermore, the important process parameter, work function variations on transfer characteristics of the device is analyzed. Moreover, the analyses tell that, for sub $-7 \mathrm{~nm}$, the NSFET is a potential device for high performance and good logic applications.

\section{Introduction}

From the past five decades, the semiconductor industry has taken major steps in designing to improve the performance of semiconductor devices. This possibility is achieved by continuous downscaling of the device dimensions. By decreasing the device dimensions, the number of transistors increased on-chip which in turn increased the performance of the device. According to Moor's Law for every eighteen months, there is a two-fold increase in the number of transistors. This device downscaling has advantages like less power consumption, high speed and reduction in cost [1-7]. The device dimensions are reduced in every successive technological node. For every technological node, the channel length is reduced and is now reached atomic dimensions. The distance between the source and drain will decrease as the channel length decreases and they are in close proximity with each other [8-9]. As a consequence of this, the lateral electric field from the source and drain starts affecting the channel in addition to the vertical electric field from the gate. This phenomenon introduces the disadvantageous short channel effects (SCEs) in orthodox MOSFETs. As the gate length is decreasing the gate loses its control over the channel which gives scope for Short Channel Effects (SCEs) and leakage currents. Some of the short channel effects are threshold voltage roll-off, drain induced barrier lowering (DIBL), hot electron effects etc [10-13]. To alleviate these SCEs the gate control on the channel should be enhanced and to achieve this, devices with multiple gates are one of the viable options. Double Gate, Trigate, FinFET and Gate All Around (GAA) nanosheet and nanowire structures are the example of multigate devices [14-23].

To get more drive current, FinFETs need tall and thin fins which increase the cost and complexity of fabrication of the device [24]. In GAA structures the gate wraps around the channel in all directions and offers potential control over the channel by the gate. However, it is noticed that the GAA stacked nanowire 
performance is degraded by the surface roughness factor [25]. For sub $7 \mathrm{~nm}$ technology node and beyond the GAA nanosheet structures emerged as potential contenders for conventional FinFETs because of their excellent control on channel region and are successors of FinFETs due to better leakage control and high current drive capability. Also, nanosheet FETs are immune to short channel effects because of their good electrostatic control over channel [26]. Compared to nanowire and FinFET structures, nanosheet FETs have shown superior electrostatic performance on channel [27]. By introducing minimal changes to the fabrication of FinFET, nanosheets can be fabricated; a five-stack nanowire FET is fabricated and presented in the literature [28]. Compared to FinFET or stacked nanowires, single stack nanosheet offers greater intrinsic performance for any sheet width. Moreover, nanosheets also have a greater effective width in a given footprint, making them better at driving capacitive loads [29].

So, in this paper, vertically stacked GAA NSFET containing two nanosheets is designed and simulated for performance evaluation. The gate leakage can be reduced further by introducing high-k materials in the gate stack. In this script, the simulation of SOI NSFET is done by using a high- $k$ gate stack to maintain superior electrostatic gate control at $300 \mathrm{~K}$. The rest of the document is structured as follows. Section 2 presents the device parameters description and physical models incorporated for simulation. The performance dependence on various parameters is analysed in section 3 , Section 4 concludes and gives a summary of this paper.

\section{Nsfet Structure And Simulation Procedure}

The device structure of the NSFET is generated by using the Cogenda Genius 3D TCAD simulator [30]. The 3D NSFET with gate length $\left(L_{G}\right)$ of $16 \mathrm{~nm}$, each sheet thickness of $5 \mathrm{~nm}$, and sheet width of $10 \mathrm{~nm}$ is generated and simulated. The n-type source/drain and p-type channel regions are doped with a doping concentration of $10^{20} \mathrm{~cm}^{-3}$ and $10^{15} \mathrm{~cm}^{-3}$ respectively. For a high-k gate stack, to get an effective oxide thickness (EOT) of $0.78 \mathrm{~nm}$, thickness values of $0.5 \mathrm{~nm}$ and $1.5 \mathrm{~nm}$ are considered for $\mathrm{SiO}_{2}$ and $\mathrm{HfO}_{2}$ respectively. To ensure better electrostatic integrity, the EOT of $0.78 \mathrm{~nm}$ and gate work function of $4.6 \mathrm{eV}$ are taken for all simulations. To improve the subthreshold behaviour, a nitride spacer with a length of 5 $\mathrm{nm}$ is maintained throughout the simulations [31].

Fig. 1(a) depicts the 2-D NSFET with Nitride spacer. The 3-D view of NSFET with stacked nanosheets is shown in Fig. 1(b). 2-D view of NSFET with nanosheet thickness $\left(N_{T}\right)$ and width $\left(N_{W}\right)$ is depicted in Fig. $1(\mathrm{c})$.

The device is well calibrated using [29, 32]. The models incorporated for simulation are as follows. To account for higher doping concentrations, Fermi Dirac statistics are involved. Since thinner nanosheets are used, quantum models are considered by taking density gradient into account for quantum effects. To consider the effects of generation and recombination of carriers, the Shockley-Read-Hall (SRH) recombination model is involved. To account for the scattering phenomena like acoustic phonons and 
surface roughness, the Lombardi mobility model is included. The models used for simulations are calibrated with experimental results and the transfer characteristics are presented in Fig. 2. Table 1 gives the information of different parameters considered for simulation.

Table .1: Device parameters used in simulation

\begin{tabular}{|ll|}
\hline Device Parameter & Value \\
\hline Nanosheet thickness $(\mathrm{nm})$ & $5-9$ \\
\hline Nanosheet width $(\mathrm{nm})$ & $10-50$ \\
\hline Gate length $(\mathrm{nm})$ & 16 \\
\hline Source/Drain doping $\left(\mathrm{cm}^{-3}\right)$ & $10^{20}$ \\
\hline Channel doping $\left(\mathrm{cm}^{-3}\right)$ & $10^{15}$ \\
\hline EOT $(\mathrm{nm})$ & 0.78 \\
\hline Spacer dielectric & Nitride \\
\hline Source/Drain length $(\mathrm{nm})$ & 12 \\
\hline Underlap spacer length $(\mathrm{nm})$ & 5 \\
\hline Work function of gate $(\mathrm{eV})$ & 4.6 \\
\hline Height of the gate $(\mathrm{nm})$ & 60 \\
\hline
\end{tabular}

\section{Result Analysis}

In this section, DC characteristics are presented for NSFET. nanosheet thickness is varied in the range of 5 $\mathrm{nm}$ to $9 \mathrm{~nm}$ and width is varied from $10 \mathrm{~nm}$ to $50 \mathrm{~nm}$ to analyse the performance dependency on the geometry of NSFET. Section 3.1 discusses the various DC parameters and section 3.2 deals with different DC parameters for various gate length considerations of NSFET. Temperature and work function dependency on NSFET are shown in section 3.3.

\subsection{Impact on DC performance of NSFET with geometric variations}

The DC performance of NSFET is analysed by changing the nanosheet physical dimensions like width and thickness. The width is varied from $10 \mathrm{~nm}$ to $50 \mathrm{~nm}$ and thickness is varied from $5 \mathrm{~nm}$ to $9 \mathrm{~nm}$. In this section, the key performance parameters like on current, off current, switching ratio, threshold voltage $\left(\mathrm{V}_{\mathrm{th}}\right), \mathrm{DIBL}$, and $\mathrm{SS}$ are discussed. 
The Contour plot of the potential distribution of NSFET is shown in Fig. 3(a). It can be observed that the potential distribution at the drain side is high compared to the source side. Also, it can be clearly seen that the drain potential impact on the channel is reduced because of the spacer. Fig. 3(b) depicts transfer characteristics in both linear and log scales for various widths of nanosheet at $V_{D S}=0.7 \mathrm{~V}$ for constant nanosheet thickness of $5 \mathrm{~nm}$.

Channel width controls the amount of flow of current through the NSFET. Each sheet width is varied in the range of 10-50 $\mathrm{nm}$ and thickness varied in the range of 5-9 $\mathrm{nm}$ to study its impact on DC metrics of NSFET a fixed gate length $L_{G}=16 \mathrm{~nm}$.

The on current is directly proportional to the effective width of the channel, which leads to an increment in the on current as the width of the sheet increases. From Fig. 4 (a), it can be seen that the maximum increment of $36 \%$ in on current is observed when the thickness is increased from $5 \mathrm{~nm}$ to $9 \mathrm{~nm}$ at nanosheet width of $10 \mathrm{~nm}$. It is observed that for larger widths of nanosheet, higher $\mathrm{I}_{\mathrm{ON}}$ is obtained due to the increment of effective width of nanosheet. On the other hand, in addition to the increment in on current, the increment in the off current also observed. As the width of the channel increases, the gate loses its control on the channel and leads to more leakages in the device. These leakages will increase the off current of the device. Moreover, the increment in off current is observed for higher thickness values of nanosheet because of reduction in potential barrier height, conduction band energy in the channel for off state condition [31].

For thinner nanosheets off state current can be reduced, however, the slight reduction in the on current is observed because of the mobility degradation due to enhancement in the perpendicular electric field [31]. These results are shown in Fig .4(b). The maximum increment of 20x in off current is observed for thickness ranging from $5 \mathrm{~nm}$ to $9 \mathrm{~nm}$ at nanosheet width of $50 \mathrm{~nm}$.

Fig .5(a) shows $\mathrm{I}_{\mathrm{ON}} / \mathrm{I}_{\mathrm{OFF}}$ and SS values for different widths of nanosheet. As the nanosheet width increases from $10 \mathrm{~nm}$ to $50 \mathrm{~nm}$, the switching ratio followed decreasing manner because of the raise in off current. Moreover, as the width ranging from $10 \mathrm{~nm}$ to $50 \mathrm{~nm}$, there is a decrement of $71 \%$ and $94 \%$ in switching ratio is observed for thickness values of $5 \mathrm{~nm}$ and $9 \mathrm{~nm}$ respectively. It can be concluded that the switching performance of the device gets deteriorated with the increment in geometrical values.

Sub-threshold swing (SS) is an important metric for device application in logic circuits. The SS is defined as the change in the gate voltage needed to get a decade change in drain current. The Sub-threshold swing (SS) can be calculated by using the formula (1)

$$
S S=\left(\frac{\partial \log _{10} I_{D}}{\partial V_{G S}}\right)^{-1}
$$

From Fig .5(a), it can be seen that SS followed an increasing manner since the gate loses its control over the channel for higher widths. Furthermore, as the width ranging from $10 \mathrm{~nm}$ to $50 \mathrm{~nm}$, there is an increment of $1.6 \%$ and $8.5 \%$ in SS is observed for thickness values of $5 \mathrm{~nm}$ and $9 \mathrm{~nm}$ respectively. Since a 
lower value of SS is preferred for good sub threshold performance, nanosheet having width and height of $10 \mathrm{~nm}$ and $5 \mathrm{~nm}$ respectively outperforms with SS of $62.5 \mathrm{mV} / \mathrm{dec}$, which is near to ideal SS.

Fig .5(b) depicts DIBL and $\mathrm{V}_{\mathrm{th}}$ for different widths of nanosheet. The threshold voltage is extracted by using the constant current method at $100 \times \mathrm{W}_{\text {eff }} / \mathrm{L}_{\mathrm{G}}$. Where $\mathrm{W}_{\text {eff }}$ is given by $\mathrm{n} \times(2 \times(T+W))$, where $T$ and $\mathrm{W}$ are the thickness and the width of the nanosheet respectively. The term $\mathrm{n}$ indicates the number of vertically stacked nanosheets [31-33]. As the thickness and width of the nanosheet are increasing, from Fig .5(b), it can be seen that the threshold voltage is reducing because of SCEs. Furthermore, there is a threshold voltage roll-off of $5.3 \%$ and $8 \%$ is observed for thickness values of $5 \mathrm{~nm}$ and $9 \mathrm{~nm}$ respectively when the nanosheet width is varied from $10 \mathrm{~nm}$ to $50 \mathrm{~nm}$. It can be seen that the $V_{\mathrm{th}}$ roll-off is more for higher thickness values of nanosheet.

Drain induced barrier lowering (DIBL) is also one of the crucial sub-threshold performance metrics and it should be as low as possible for good performance of the device. The DIBL is computed by using the formula (2).

$$
D I B L=\frac{V_{t l i n}-V_{t s a t}}{V_{D s a t}-V_{D l i n}}(2)
$$

Here, $V_{\text {tlin }}$ is the threshold voltage extracted at linear supply voltage, $V_{\text {Dlin }}=0.04 \mathrm{~V}$ and $\mathrm{V}_{\text {tsat }}$ is threshold voltage extracted at saturation supply voltage, $V_{\text {Dsat }}=0.7 \mathrm{~V}$. It can be observed that, as the nanosheet dimensions are increasing, the DIBL gets increasing. Moreover, as the nanosheet width increased from 10 $\mathrm{nm}$ to $50 \mathrm{~nm}$, there is an increment of $24 \%$ and $30 \%$ in DIBL observed for nanosheet thickness values of 5 $\mathrm{nm}$ and $9 \mathrm{~nm}$ respectively. The DIBL can be lowered by incorporating the nanosheets which are having smaller widths and thickness values. It can be concluded that DIBL is sensitive towards width and thickness variations.

\subsection{Impact on DC performance of NSFET with scaling.}

In this section, the impact of scaling on the DC performance of NSFET is analysed for sub - $7 \mathrm{~nm}$ technology nodes. The gate length is downscaled from $20 \mathrm{~nm}$ to $5 \mathrm{~nm}$ and the results are depicted in Fig. 6(a). The transfer characteristics are obtained by fixing the thickness to $5 \mathrm{~nm}$ and width to $10 \mathrm{~nm}$ for the nanosheet.

From Fig. 6(b), it is observed that as the channel length decreases, the on current is increased because the distance between source and drain is decreased. However, the OFF current also increased as the channel length decreases due to the SCEs.

Figure 7(a) depicts the switching ratio and SS as a function of the gate length. It can be seen that as the channel length is decreasing, the switching ratio has deteriorated. However, even at $L_{G}$ of $5 \mathrm{~nm}$, the switching ratio of $10^{6}$ is maintained which ensures that NSFET is a suitable candidate for good logic applications and continued scaling [34]. Moreover, as the gate length decreasing, the SS is increased and 
there is an increment of $31 \%$ is observed as the gate length is scaled from $20 \mathrm{~nm}$ to $5 \mathrm{~nm}$. The increment in SS is due to an increment in leakage currents. DIBL and $V_{\text {th }}$ are shown in Fig .7(b). DIBL is another parameter that affects severely with scaling. As the gate length decreases, the impact of drain potential is more on the channel and leads to the increment of DIBL. From Figure 7(b), it can be seen that the DIBL is increased and a growth rate of $339 \%$ is observed as the gate length ranging from $20 \mathrm{~nm}$ to $5 \mathrm{~nm}$. The threshold voltage variations as a function of gate length are depicted in Figure $7(b)$. The deterioration in threshold voltage is observed as the gate length is increasing due to the threshold voltage roll-off. As the gate length decreased from $20 \mathrm{~nm}$ to $10 \mathrm{~nm}$, a reduction of $1.9 \%$ in threshold voltage is observed, whereas from $10 \mathrm{~nm}$ to $5 \mathrm{~nm}$, a reduction of $7 \%$ is observed. It is obvious that for sub $-10 \mathrm{~nm}$, the threshold voltage roll-off is more due to increased SCEs.

\subsection{Impact of temperature and work function variations on NSFET}

Nanoscale transistors are used in a wide variety of fields like communication, automobiles, medical equipment, analog and digital integrated circuits, sensing applications and power electronics. As per the requirement, the nano transistors are used at different temperatures and are crucial to analyze the performance of transistors at various ranges of temperatures [35].

Temperature dependency on drain current as a function of gate voltage is plotted in Fig. 8(a). The temperature is varied in the range of $-43^{\circ} \mathrm{C}$ to $127^{\circ} \mathrm{C}$. It is very much required to bias these circuits so that the performance or V-I characteristics are insensitive or independent towards temperature variations. IC designers are very much interested to know this inflection point of temperature. This biasing point is called the temperature compensation point (TCP) [36]. From Fig. 8(a), the TCP is observed at $\mathrm{V}_{\mathrm{GS}}=0.55 \mathrm{~V}$ and $I_{D}=3.48 \times 10^{-6}$ A. From Fig. $8(b)$, it is clear that as the temperature increases, increment in off currents is observed because of the dominance of impurity scattering. Moreover, a slight decrement in on current is observed with raise in temperature due to the mobility reduction.

$\mathrm{I}_{\mathrm{ON}} / \mathrm{I}_{\mathrm{OFF}}$ and SS variations as a function of temperature are presented in Fig. 9(a). It is observed that there is deterioration in the switching ratio as the temperature increases because of the significant improvement in off current. Furthermore, the value of SS is less at low temperatures and ensures faster operation of the device [37]. Fig. 9(b) depicts DIBL and $V_{\text {th }}$ variations with respect to temperature and is observed that as temperature increases, the rise in DIBL is observed because of the decrement in threshold voltage. Furthermore, threshold voltage deteriorated with the rise in temperature due to scattering phenomena [38]. A Threshold voltage roll-off of $9.7 \%$ is observed as temperature increased from $-43^{\circ} \mathrm{C}$ to $127^{\circ} \mathrm{C}$.

Work function is one of the important process parameter which plays a detrimental role in turning on and off of a device. Higher gate work function ensures that the device is fully depleted rapidly and that the device performs better in the off state [39]. 
Work function impact on drain current as a function of gate voltage is shown in Fig. 10(a). The work function of the device is varied from $4.3 \mathrm{eV}$ to $4.7 \mathrm{eV}$. From Fig. 10(b) it can be inferred that for higher work function of the device, along with the deterioration in on current, significant reduction in off current is observed. An Increment in work function lowers the tunnelling between gate and channel and also between gate and drain/source [40], which helps to reduce the leakage current.

Switching ratio variations with respect to work function are shown in Fig. 11 (a). The switching ratio improved significantly with the rise in work function due to a huge reduction in off current. With the increment of work function, the SS gets decremented and ensures good sub threshold performance. Fig. 11(b) depicts the DIBL and threshold voltage variations as a function of the work function. DIBL is decreasing with the rise in work function and lesser DIBL indicates that electrostatic integrity of the gate is more on the device and less sensitive to drain voltage variations. A minimum DIBL of $30.30 \mathrm{mV} / \mathrm{V}$ is obtained for work function of $4.7 \mathrm{eV}$ and there is a decrement of $28.6 \%$ in DIBL is observed when the work function varied from $4.3 \mathrm{eV}$ to $4.7 \mathrm{eV}$. However, there is a huge decrement in threshold voltage with the fall in work function of the device. There is a decrement of $3.9 \mathrm{x}$ in threshold voltage as the work function is decreased from $4.7 \mathrm{eV}$ to $4.3 \mathrm{eV}$, and leads to more SCEs. Moreover, the study tells that the devices with more work function will have high threshold voltages and makes the device sluggish.

\section{Conclusion}

In this paper, the detailed DC performance of 3D vertically stacked NSFET is analyzed by varying the thickness and width of the nanosheet. It is observed that there is an increment in both on current and off current as the width and thickness values are increased. However, the switching ratio, DIBL, and SS are degraded as the dimensions of NSFET are increasing. Moreover, the performance of NSFET is analysed with scaling of gate length to ensure the device feasibility for lower technology nodes. Also, the temperature analysis is performed for a wide range of temperatures to identify the TCP point, which is more essential for biasing the device in various real time applications. Finally, the impact of work function on device transfer characteristics is analysed. These results will give deep insights into the performance of NSFET with different device parametric variations.

\section{Declarations}

\section{Author Contributions}

N. Aruna Kumari: Methodology, Writing- Original draft preparation, Formal Analysis, Software, Investigation, Visualization, Data Curation.

P. Prithvi: Supervision.

Financial interests: The authors declare they have no financial interests. 
Funding: No Funding Received.

Conflict of Interest: The author has no conflicts of interest to declare that are relevant to the content of this article.

\section{Compliance with Ethical Standards:}

- The contents of this manuscript are not now under consideration for publication elsewhere;

- The contents of this manuscript have not been copyrighted or published previously

- The contents of this manuscript will not be copyrighted, submitted, or published elsewhere, while acceptance by the Journal is under consideration.

Availability of data and material: Not applicable.

Consent to Participate: Not applicable.

Consent for Publication: Not applicable.

Acknowledgements: The authors thank to the department of Electronics and Communications Engineering, NIT Warangal for providing the TCAD Tools.

\section{References}

1. Zhang, J. G. Fossum, L. Mathew and Y. Du, Physical insights regarding design and performance of independent-gate FinFETs, IEEE Trans. Electron Devices, 52 (10) (2005) 2198-2206.

2. B. Sreenivasulu, V. Narendar, Performance improvement of spacer engineered n-type SOI FinFET at 3nm gate length, AEU - International Journal of Electronics and Communications, 137, 2021, 153803.

3. Tayal and A. Nandi, Study of 6T SRAM cell using high-K gate dielectric based junctionless silicon nanotube FET, Superlattices and Microstructures,2017, DOI: 10.1016/j.spmi.2017.08.061

4. Ghosh, P, Bhowmick, B. Effect of temperature in selective buried oxide TFET in the presence of trap and its RF analysis. Int J RF Microw Comput Aided Eng. 30: e22269 (2020).

5. Baral, P.K. Singh, S. Kumar, S. Chander, S. Jit, Ultrathin body nanowire hetero dielectric stacked asymmetric halo doped junctionless accumulation mode MOSFET for enhanced electrical characteristics and negative bias stability, Superlattice. Microst. 138 (2019), https://doi.org/10.1016/j.spmi.2019.106364

6. Vishal Narula, Amit Saini \& Mohit Agarwal (2021) Correlation of Core Thickness and Core Doping with Gate \& Spacer Dielectric in Rectangular Core Shell Double Gate Junctionless Transistor, IETE Journal of Research, DOI: 10.1080/03772063.2021.1946437

7. Kumar, B., Chaujar, R. Numerical Study of JAM-GS-GAA FinFET: A Fin Aspect Ratio Optimization for Upgraded Analog and Intermodulation Distortion Performance. Silicon (2021). https://doi.org/10.1007/s12633-021-01395-8 
8. Sreenivasulu, V.B., Narendar, V. A Comprehensive Analysis of Junctionless Tri-gate (TG) FinFET Towards Low-power and High-frequency Applications at 5-nm Gate Length. Silicon (2021).

9. Roy Barman, K., Baishya, S. Performance analysis of vertical super-thin body (VSTB) FET and its characteristics in presence of noise. Appl. Phys. A 125, 401 (2019). https://doi.org/10.1007/s00339019-2682-x

10. Barman, K.R., Baishya, S. An Insight into the DC and Analog/RF Response of a Junctionless Vertical Super-Thin Body FET towards High-K Gate Dielectrics. Silicon (2021). https://doi.org/10.1007/s12633-021-01393-w

11. Kale, S., Chandu, M.S. Dual Metal Gate Dielectric Engineered Dopant Segregated Schottky Barrier MOSFET With Reduction in Ambipolar Current. Silicon (2021). https://doi.org/10.1007/s12633-02000921-4

12. Crupi G, Caddemi A, Schreurs DMM-P, Wiatr W, Mercha A.Microwave noise modelling of FinFETs. Solid-State Electron.2011;56:18-22

13. Singh, R., Kaim, S., MedhaShree, R. et al. Dielectric Engineered Schottky Barrier MOSFET for Biosensor Applications: Proposal and Investigation. Silicon (2021). https://doi.org/10.1007/s12633021-01191-4

14. Das, D, Baishya, S, Chakraborty, U. Impact of temperature on RF characteristics and electrical noise analysis of an L-shaped gate tunnel FET with hetero-stacked source configuration. Int J RF Microw Comput Aided Eng, 30: e22310 (2020).

15. saha, B. Bhowmick, S. Baishya, Study on impact of ferroelctric layer thickness on RF/analog and linearity parameters in ferroelctric-FinFET. Int J RF Microw Comput Aided Eng, (2021)

16. He, J. Fronheiser, P. Zhao, Z. Hu, S. Uppal, X. Wu, Y. Hu, R. Sporer, L. Qin, R. Krishnan, E. M. Bazizi, R. Carter, K. Tabakman, A. K. Jha, H. Yu, O. Hu, D. Choi, J. G. Lee, S. B. Samavedam and D. K. Sohn, "Impact of aggressive !n width scaling on Infet device characteristics," IEEE International Electron Devices Meeting (IEDM), San Francisco, CA, USA, 2017. DOI: 10.1109/IEDM.2017.8268427

17. Khalil Tamersit. Sub-10 nm junctionless carbon nanotube field-effect transistors with improved performance, AEU - International Journal of Electronics and Communications. 2020; 124:153354

18. Katti, N. DasGupta, and A. DasGupta, "Threshold voltage model for mesaisolated small geometry fully depleted SOI MOSFETs based on analytical solution of 3-D Poisson's equation." IEEE Trans. Electron Devices, 51, 1169 (2004)

19. Kumar B, Chaujar R (2021) Analog and RF performance evaluation of Junctionless accumulation mode (JAM) gate stack gate allaround (GS-GAA) FinFET. Silicon.https://doi.org/10.1007/s12633020-00910-7

20. Gupta, N., Kumar, A. \& Chaujar, R. Design Considerations and Capacitance Dependent Parametric Assessment of Gate Metal Engineered SiNW MOSFET for ULSI Switching Applications. Silicon 12, 1501-1510 (2020). https://doi.org/10.1007/s12633-019-00246-x

21. Kumar, R and Kumar. A. Hafnium based high-k dielectric gate-stacked (GS) gate material engineered (GME) junctionless nanotube MOSFET for digital applications. Appl. Phys. 2021; A 127, 26 
22. Rajiv Ranjan Thakur and Nidhi Chaturvedi, Design, Optimization, and Analyisis of Si and GAN Nanowire FETs for 3 nm Technology, Semicond. Sci. Technol. 362021075013.

23. Sanjay, Prasad, B. \& Vohra, A. 2021 Effect of $3 \mathrm{~nm}$ gate length scaling in junctionless double surrounding gate SINT MOSFET by using triple material gate engineering. Microsyst Technol.

24. Sreenivasulu V and Narendar V. Design and Deep Insights into Sub-10 nm Spacer Engineered Junctionless FinFET for Nanoscale Applications. ECS J. Solid State Sci. Technolo. 2021; 10: 013008

25. M. Buß̧er, R. Ritzenthaler, H. Mertens, G. Eneman, A. Mocut and N. Horiguchi, "Performance Comparison of n-Type Si Nanosheets, and FinFETs by MC Device Simulation," IEEE Electron Device Lett., vol. 39, no. 11, p. 1628-1631, Nov 2018. DOI: 10.1109/LED.2018.2868379

26. Kalna, D. Nagy, A. J. García-Loureiro and N. Seoane, "3D Schrödinger Equation Quantum Corrected Monte Carlo and Drift Diffusion Simulations of Stacked Nanosheet Gate-All-Around Transistor," IWCN, Wien: Institute for Microelectronics, TU Wien, May,2019, pp. 33-35.

27. D. Kim, M. Guillorn, I. Lauer, P. Oldiges, T. Hook and M. H. Na, "Performance Trade-offs in FinFET and Gate-All-Around Device Architectures for 7nm-node and Beyond," 2015 IEEE SOI-3DSubthreshold Microelectronics Technology United Conference (S3S), Rohnert Park, CA, USA, Oct. 2015. DOI: $10.1109 /$ S3S.2015.7333521

28. Byung-Hyun Lee, Min-Ho Kang, Dae-Chul Ahn, Jun-Young Park, Tewook Bang, Seung-Bae Jeon, Jae Hur, Dongil Lee, Yang-Kyu Choi, Vertically integrated multiple nanowire field effect transistor, Nano Lett. 15 (2015) 8056-8061.

29. Loubet et al., "Stacked nanosheet gate-all-around transistor to enablescaling beyond FinFET," Proc. Symp. VLSI Technol., Jun. 2017,pp. 230-231, doi: 10.23919/VLSIT.2017.7998183.

30. Genius, 3-D Device Simulator, Version1.9.0, Reference Manual, Cogenda, Singapore, 2008.

31. Sreenivasulu, V.B., Narendar, V. Design and Temperature Assessment of Junctionless Nanosheet FET for Nanoscale Applications. Silicon (2021). https://doi.org/10.1007/s12633-021-01145-w

32. Jegadheesan, K. Sivasankaran, and A. Konar, "Impact of geometrical parameters and substrate on analog/RF performance of stackednanosheet field effect transistor," Mater. Sci. Semicond. Process., vol. 93,pp. 188-195, Apr. 2019, doi: 10.1016/j.mssp.2019.01.003

33. Min-Ju Ahn et al. Superior subthreshold characteristics of gate-all-around (GAA) p-type junctionless poly-Si nanowire transistor with ideal subthreshold slope. $2020 \mathrm{Jpn}$. J. Appl. Phys. in press https://doi.org/10.35848/1347-4065/ab9e7d

34. International technology roadmap for semiconductors (ITRS), Semicond. Ind. Assoc. 1 (2015).

35. Barman, K.R., Baishya, S. Study of Temperature Effect on Analog/RF and Linearity Performance of Dual Material Gate (DMG) Vertical Super-Thin Body (VSTB) FET. Silicon 13, 1993-2002 (2021). https://doi.org/10.1007/s12633-020-00561-8

36. Kumar, B., Chaujar, R. TCAD Temperature Analysis of Gate Stack Gate All Around (GS-GAA) FinFET for Improved RF and Wireless Performance. Silicon 13, 3741-3753 (2021). https://doi.org/10.1007/s12633-021-01040-4 
37. S.P. Wong, D.J. Frank, P.M. Solomon, C.H.J. Wann, J.J. Welser, Nanoscale CMOS, P. IEEE 87 (4) (1999) 537-570, http:// dx.doi.org/10.1109/5.752515.

38. Toan, H.L.M., Singh, S.S. \& Maity, S.K. Analysis of Temperature Effect in Quadruple Gate Nano-scale FinFET. Silicon 13, 2077-2087 (2021). https://doi.org/10.1007/s12633-020-00615-x

39. Bharath Sreenivasulu, Vadthiya Narendar, Characterization and optimization of junctionless gate-allaround vertically stacked nanowire FETs for sub- $5 \mathrm{~nm}$ technology nodes, Microelectronics Journal, 116, (2021),105214,

40. Yong-Tian Hou, Ming-Fu Li, T. Low and Dim-Lee Kwong. Metal gate work function engineering on gate leakage of MOSFETs, IEEE Transactions on Electron Devices.2004; 51(11):1783-1789

\section{Figures}

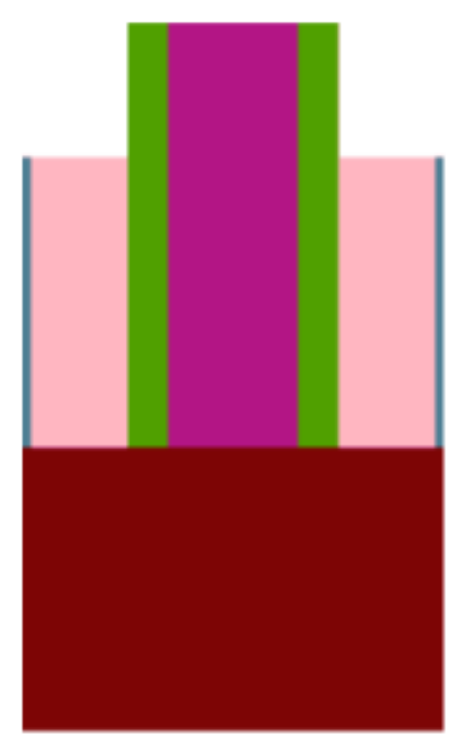

(a)

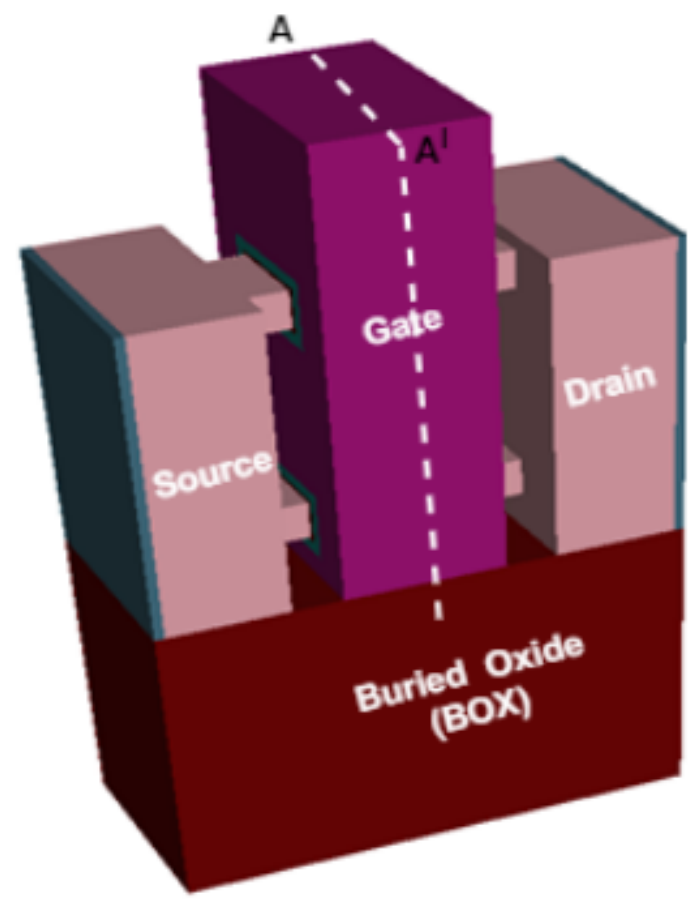

(b)

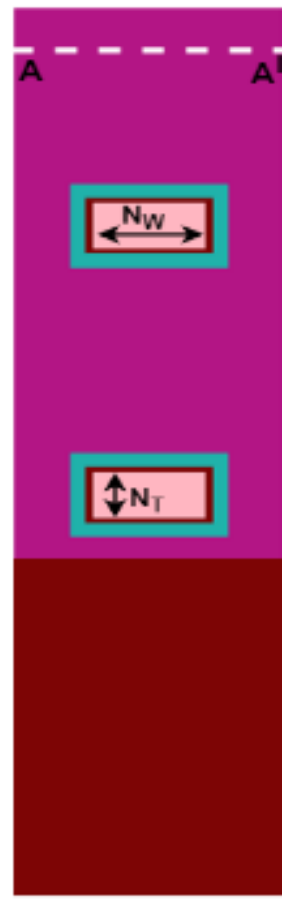

(c)

\section{Figure 1}

(a) 2-D view of NSFET with nitride spacer, (b) 3-D schematic view of NSFET and (c) 2-D cross section view of NSFET 


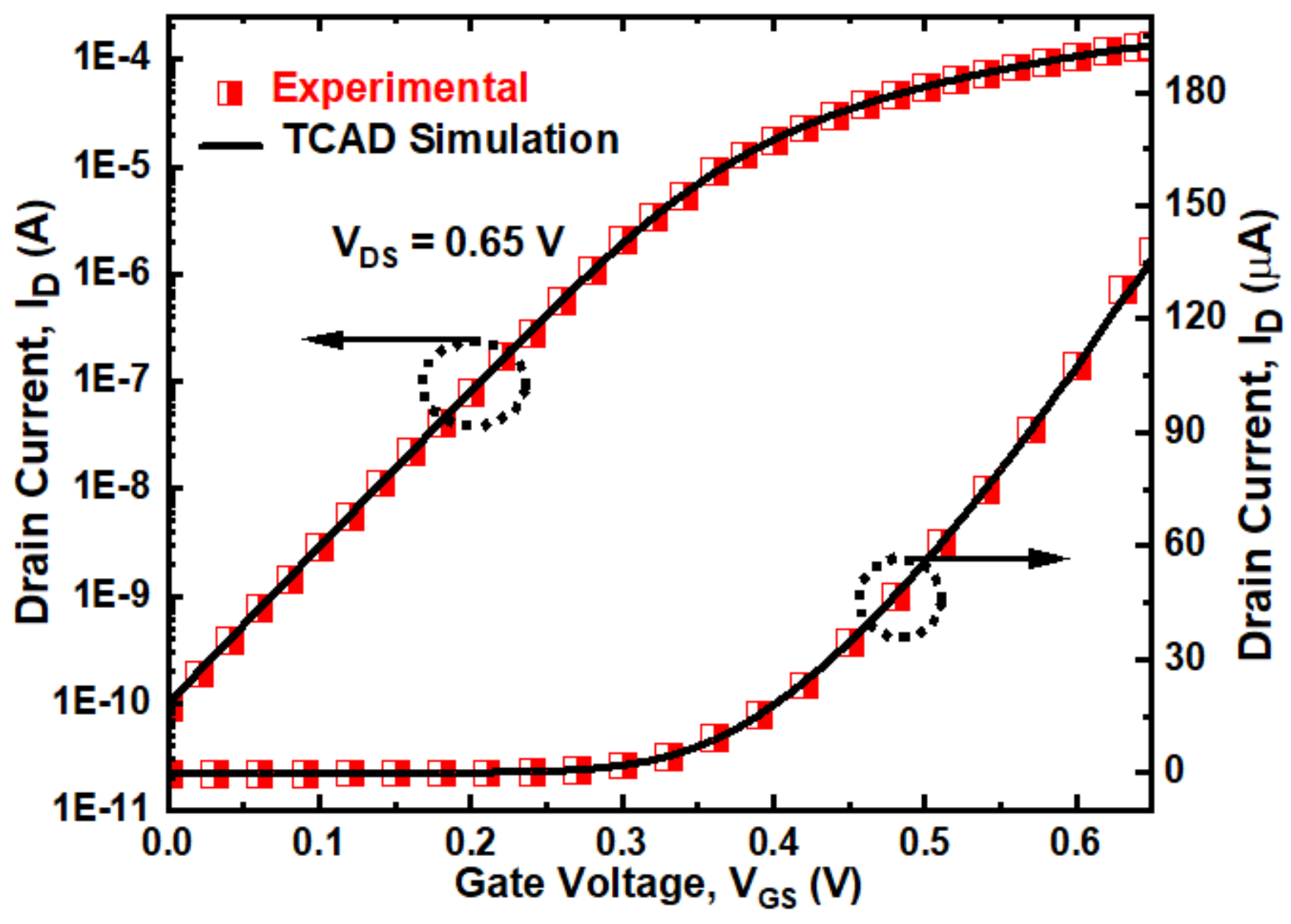

Figure 2

Calibration of simulation models with that of experimental results [27]

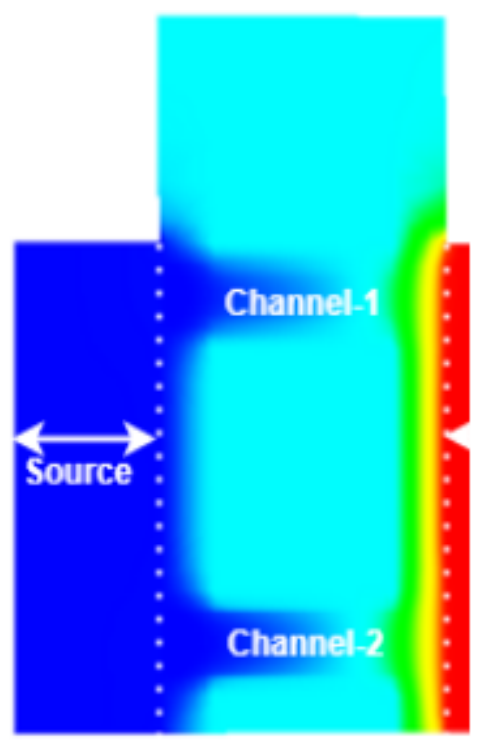

(a)

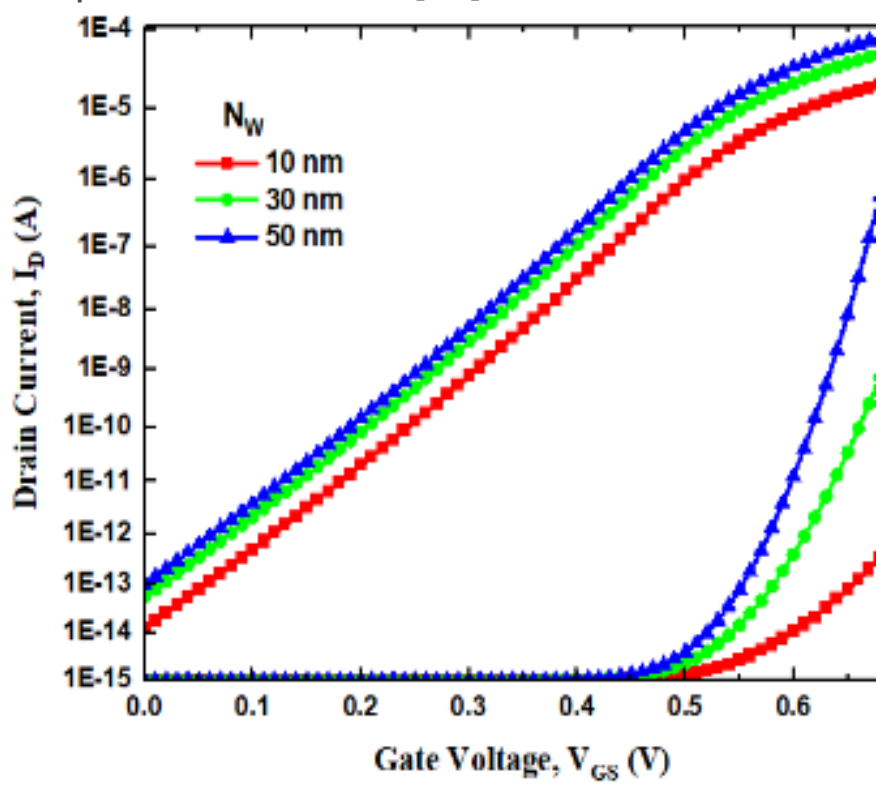

(b)

Figure 3 
(a) Contour plot of the potential distribution of NSFET in ON state (b) Transfer characteristics of NSFET in linear and log scale for VDS $=0.7 \mathrm{~V}$.

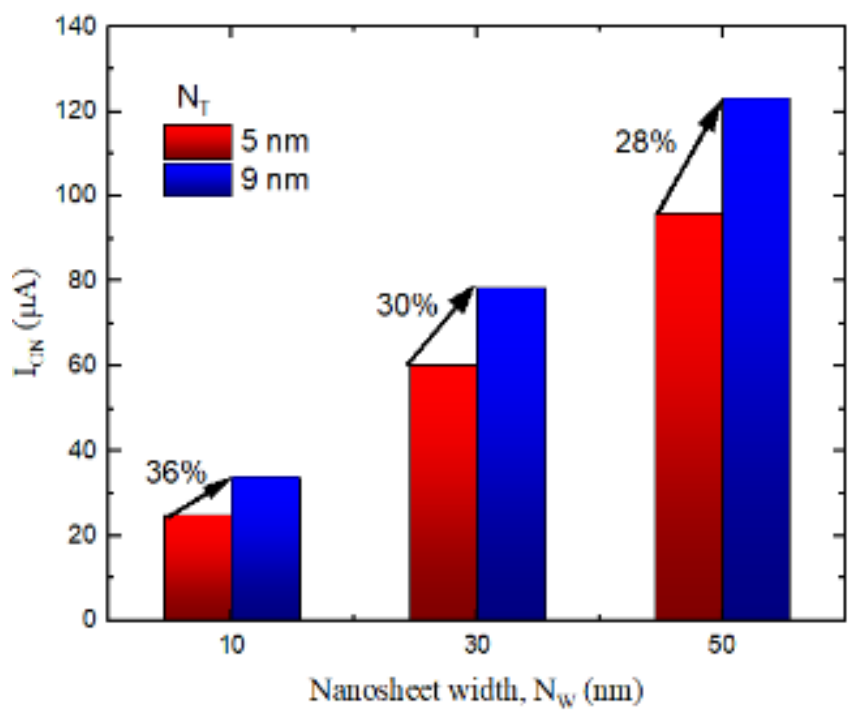

(a)

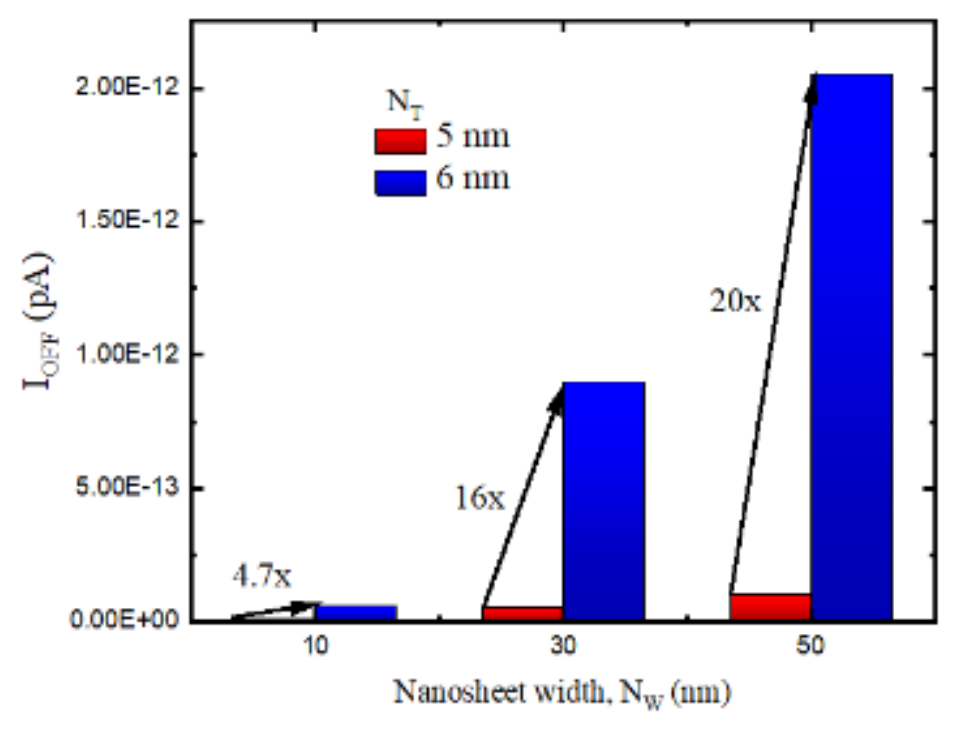

(b)

Figure 4

(a) ION and (b) IOFF as a function of NW for various NT values

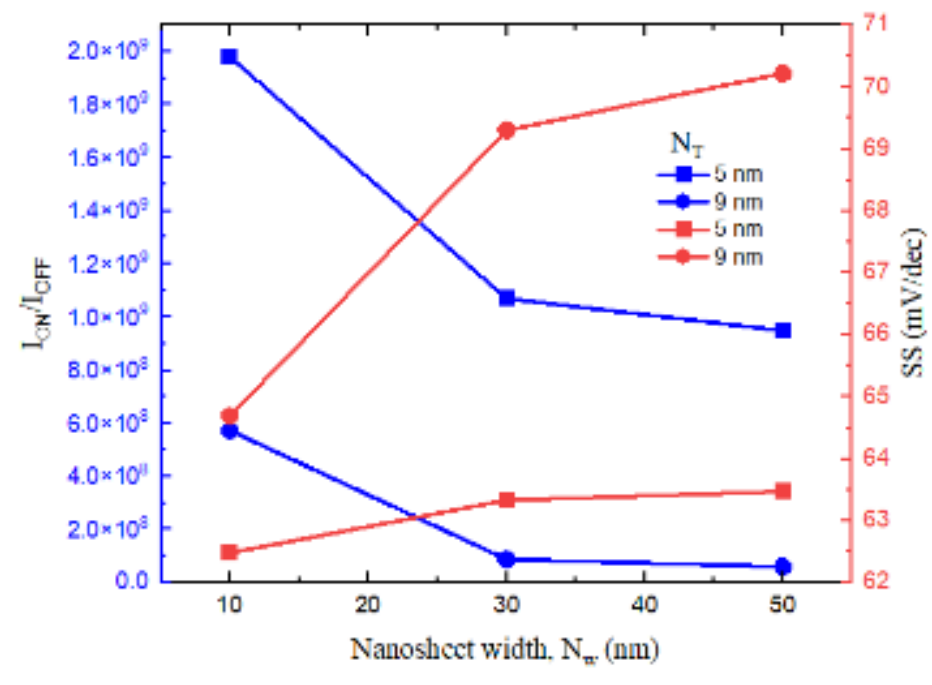

(a)

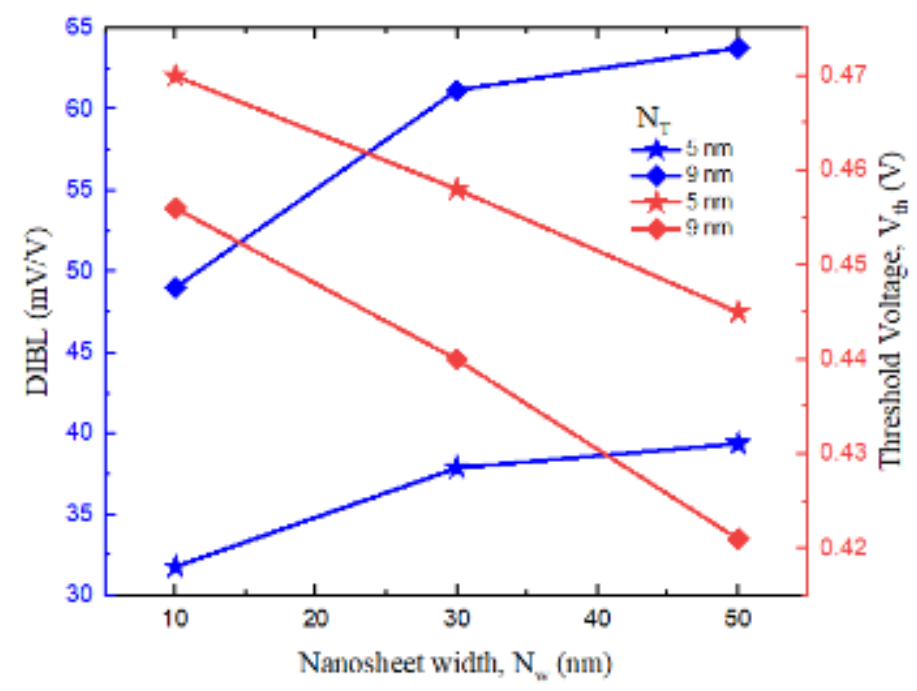

(b)

Figure 5

(a) ION/ IOFF and SS (b) DIBL and Vth as a function of NW for various NT values 


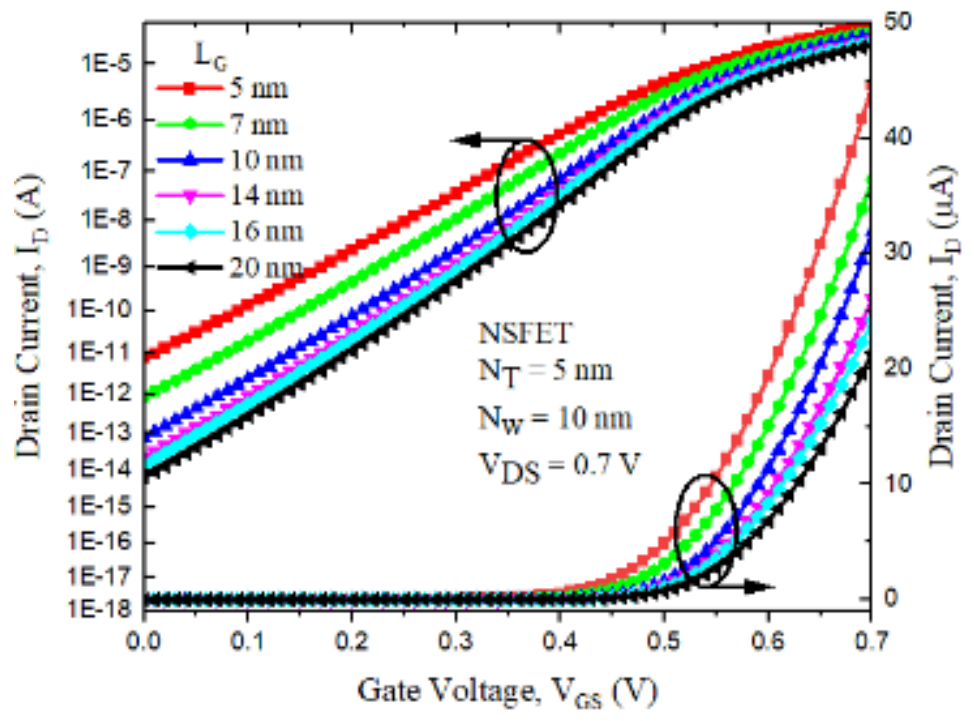

(a)

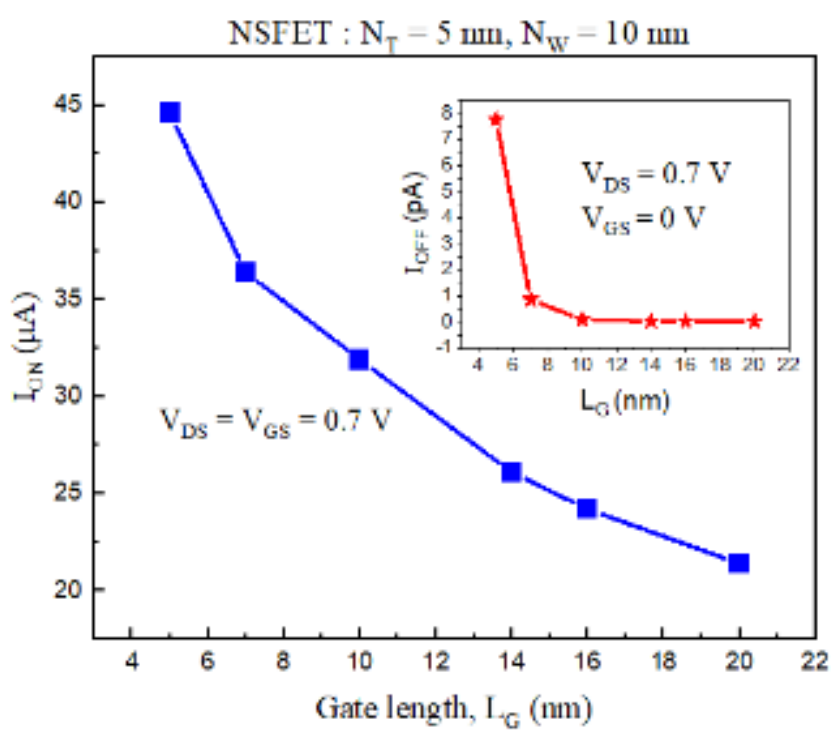

(b)

Figure 6

(a) Transfer characteristics for various LG values and (b) ION and IOFF (inset) as a function of LG

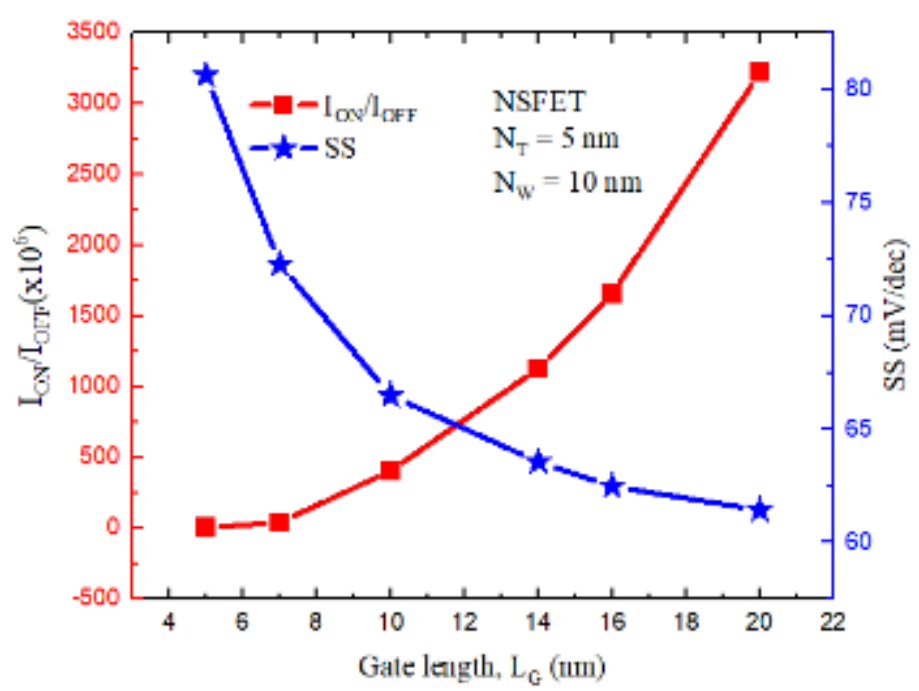

(a)

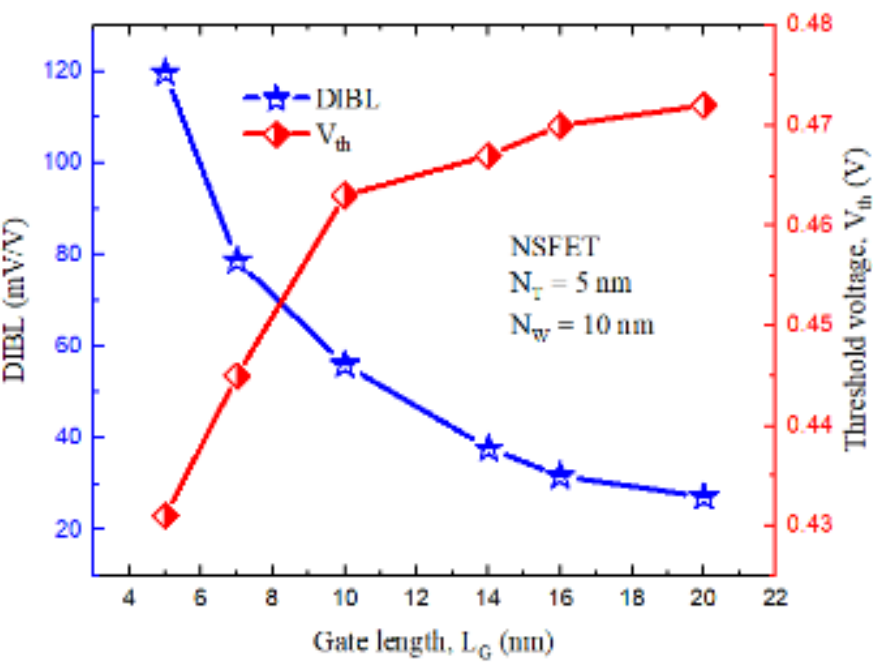

(b)

Figure 7

(a) ION/ IOFF and SS (b) DIBL and Vth as a function of LG 


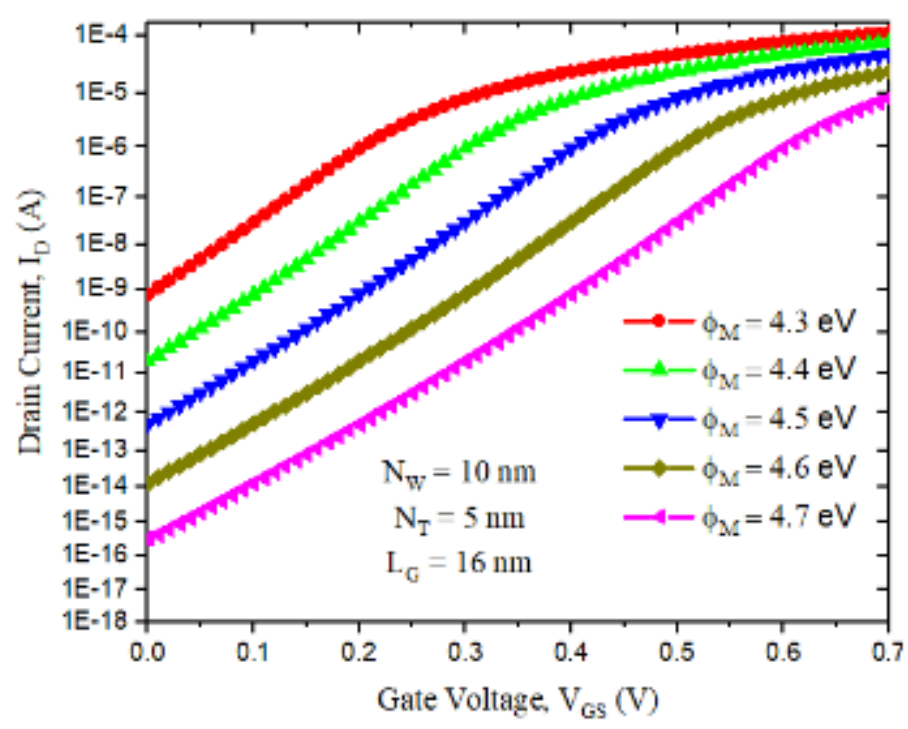

(a)

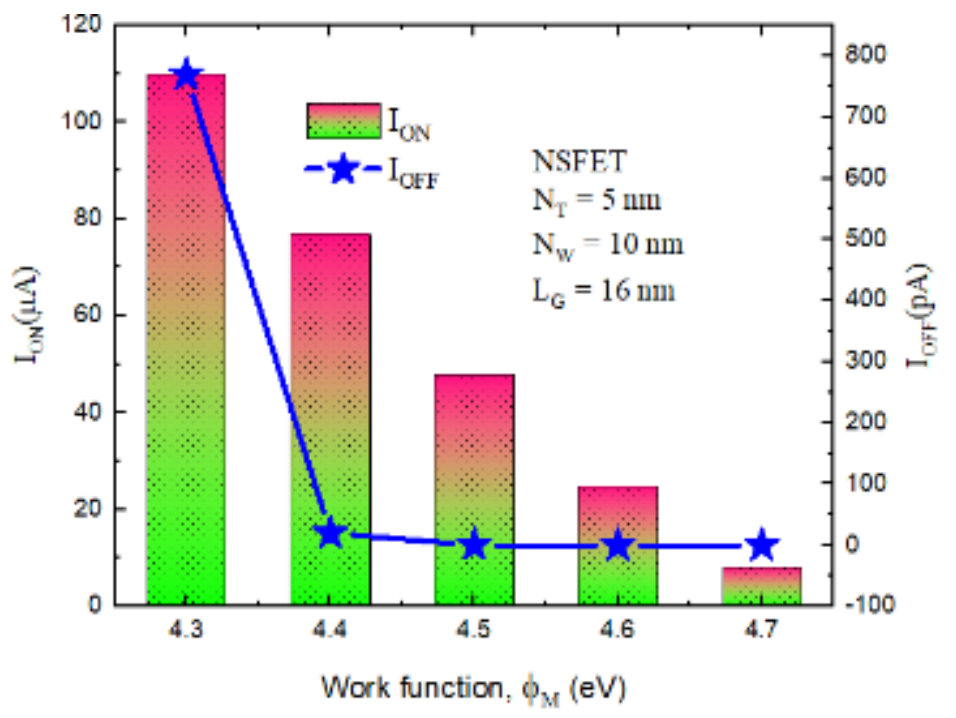

(b)

\section{Figure 8}

(a) Temperature variations (b) ION and IOFF variations as a function of VGS

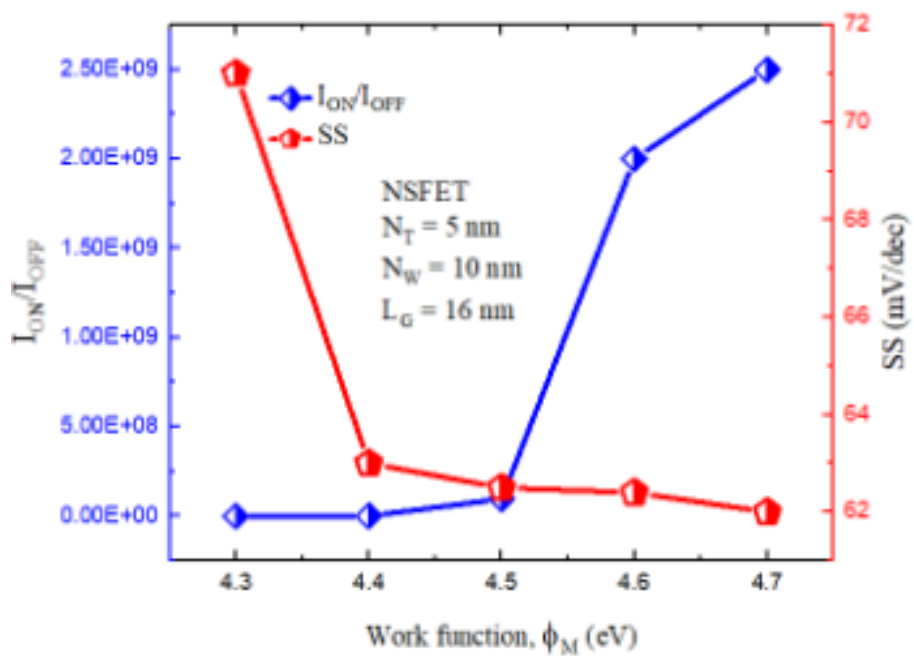

(a)

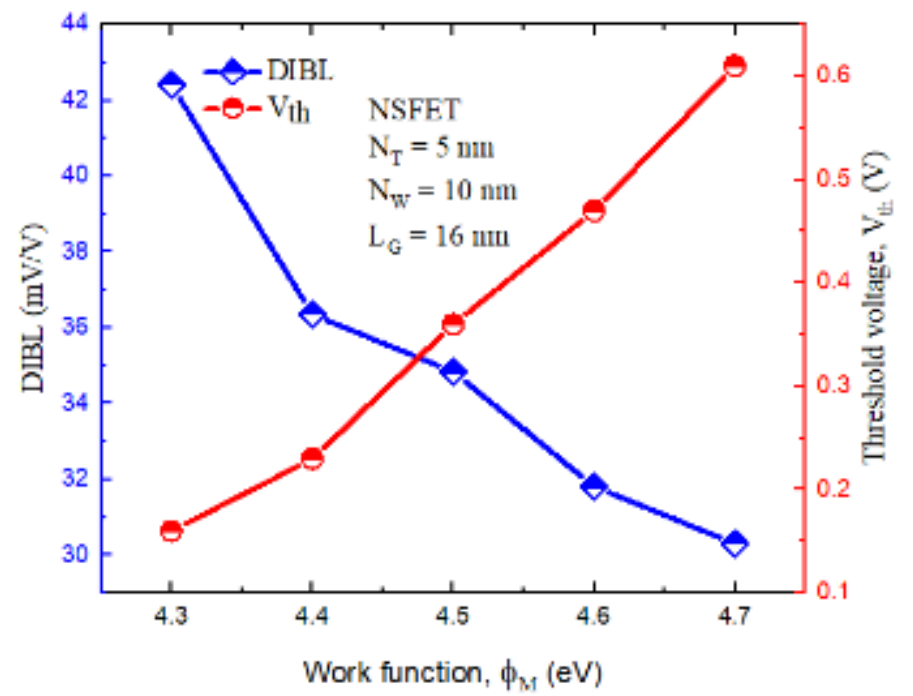

(b)

Figure 9

(a) ION/IOFF and SS (b) DIBL and Vth variations as a function of temperature 


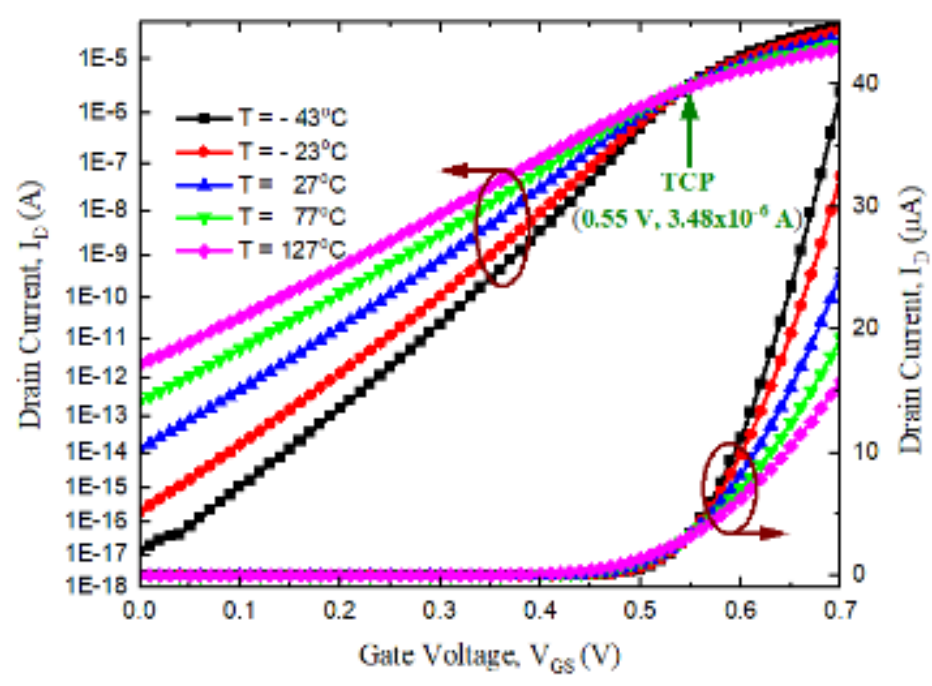

(a)

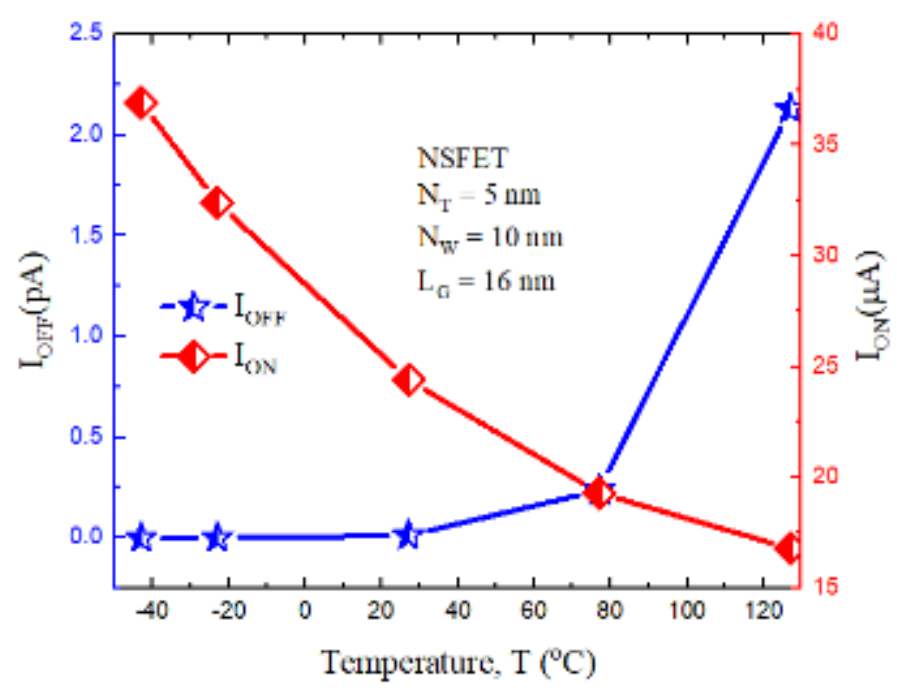

(b)

Figure 10

(a) Drain current variations with work function (b) ION and IOFF variations as a function work function

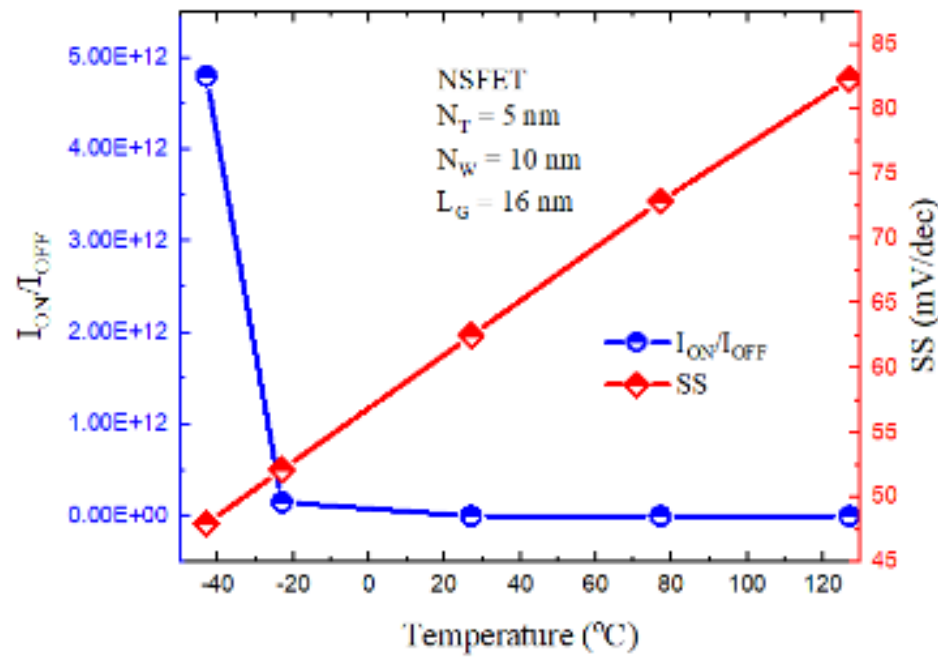

(a)

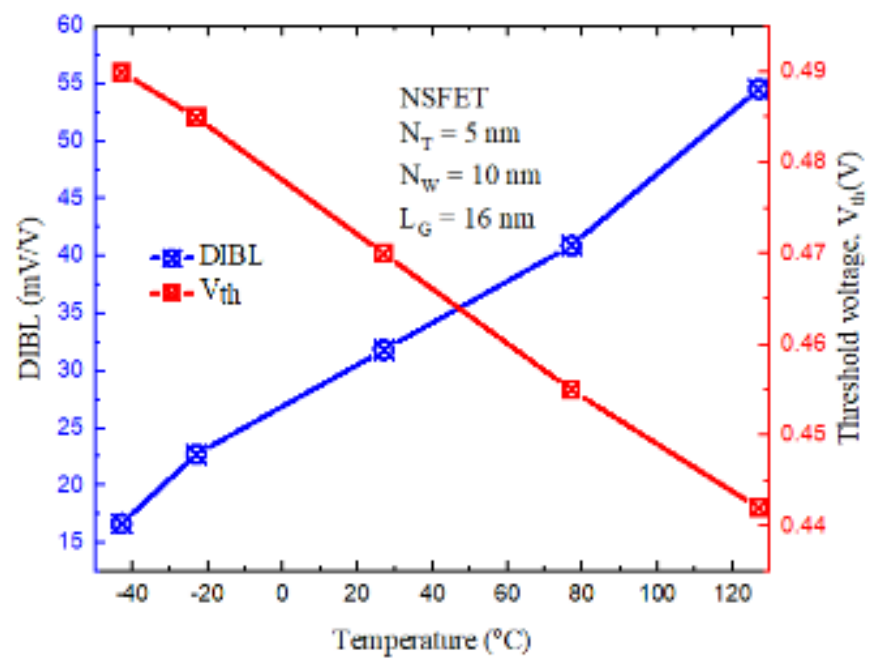

(b)

\section{Figure 11}

(a) ION/IOFF and SS (b) DIBL and Vth variations as a function of work function 